\title{
Cardiovascular disease in transsexual persons treated with cross-sex hormones: reversal of the traditional sex difference in cardiovascular disease pattern
}

\author{
Louis J Gooren ${ }^{\dagger}$, Katrien Wierckx' ${ }^{1}$ and Erik J Giltay ${ }^{2}$ \\ Emeritus, VU University Medical Center, PO Box 7057, NL-1007 MB Amsterdam, The Netherlands, \\ ${ }^{1}$ Department of Endocrinology, UZ Gent, Gent, Belgium and ${ }^{2}$ Department of Psychiatry, \\ Leiden University Medical Center, Leiden, The Netherlands \\ ${ }^{\dagger} \mathrm{L} J$ Gooren is now at 72/1, moo 1. T.Palan, A.DoiSaket, Chiang Mai 50220, Thailand
}

Correspondence should be addressed to L J Gooren

Email

louisjgooren@gmail.com

\begin{abstract}
Objective: The incidence of heart disease increases with age, but is lower in women than in men up to 75 years. A protective effect of female sex hormones or, alternatively, acceleration in male heart disease by testosterone at younger ages, could explain this sex difference. In contrast with the above, male-to-female transsexual subjects (MtoF) treated with estrogens (+ anti-androgens) show more cardiovascular pathology than female-to-male transsexual subjects (FtoM) receiving testosterone. Why MtoF suffer more frequently from cardiovascular disease than females is as yet unclear. The mode of cross-sex hormone treatment may be a factor, and, if so, it may need adaptations.

Subjects and methods: Studies in transsexual people on the effects of cross-sex hormone treatment on surrogate cardiovascular risks and on clinical endpoints were reviewed. With regard to MtoF, a parallel was sought with men with prostate cancer, undergoing androgen deprivation and estrogen administration.

Results: Exposure of FtoM to testosterone was not associated with a strong increase in cardiovascular events. Aging and pre-existing cardiovascular pathology contributed to the risk of cardiovascular disease in MtoF. Use of the synthetic biopotent compound ethinyl estradiol in a dose two to four times of oral contraceptives increased cardiovascular risk substantially. The route of administration of estrogens (oral vs transdermal) may have impacted on the risks.

Conclusion: MtoF should not be treated with oral ethinyl estradiol. Transdermal estrogens are probably safer than oral estrogens. Pre-existing cardiovascular risks should be taken into consideration when prescribing and choosing the type of estrogens in crosssex hormone administration (oral vs transdermal). In addition, risk factors, as they emerge with aging, should be addressed.

\section{Introduction}

This contribution reviews the, at first sight somewhat unexpected, finding that in transsexual subjects cardiovascular pathology is more prevalent in male-to-female transsexual subjects (MtoF) receiving treatment with estrogen (+anti-androgens, sometimes with progestational properties) than in female-to-male transsexual subjects (FtoM) receiving testosterone. This contrasts with the general population wherein, at all ages, men, at least up to about 65-75 years of age, have a survival disadvantage relative to women, usually attributed to more cardiovascular pathology in men, while the latter is also occurring at a younger age in men.

The present analysis started with the underlying assumption that cardiovascular disease patterns of MtoF (c) 2014 European Society of Endocrinology Printed in Great Britain
Published by Bioscientifica Ltd. 
treated with estrogens show similarities with those of women and, conversely, patterns of FtoM treated with testosterone similarities to men's patterns. However, this is not the case in transsexual subjects. In this contribution, we will examine a number of factors related to cross-sex hormone treatment MtoF and FtoM receive. It will be analyzed whether these factors could account for the higher prevalence of cardiovascular disease in MtoF. If so, modifications of cross-sex hormone treatment may be necessary. The sex difference in cardiovascular mortality favoring women has been observed across a diversity of human populations and also in other species (1). However, how the sex gap evolves with individual aging is still poorly understood $(2,3)$. Studies of cause-specific mortality indicate that sex differences in cardiovascular diseases are for a large part responsible for the sex gap in adult mortality and also why this gap narrows at old ages $(4,5,6)$ when women are aging and suffer increasingly frequently from heart disease. Women have a lower heart disease mortality at any age, but when they reach their midseventies, they have survived large numbers of men. Also in women heart disease mortality increases with aging and because they outnumber men at that age, the number of women dying of heart disease becomes similar to the mortality in men once they reach the age of 75 (7). In men, there is an about 10-year head start and cardiovascular disease is also more severe. The classic Kalin \& Zumoff (8) study showed a rather consistent MtoF ratio of 2:1 for fatal coronary heart disease in countries with very different rates of heart disease. Interestingly, over the last 2 decades it has become clear that men with low testosterone concentrations are high-risk individuals with regard to cardiovascular disease and mortality from all causes (9).

If it is to be believed that the sex difference in cardiovascular pathology may be related to the differences in sex steroid milieus between men and women; this protective effect with regard to cardiovascular disease in women would be expected to be lost at menopause. Postmenopausally, there is no increase in cardiovascular disease above the age-related increase. This is in contrast with the rate of breast malignancies which decline upon the postmenopausal decline of estrogen production (for review: $(10,11,12)$ ). Alternatively, acceleration in male heart disease mortality at younger ages could explain sex differences rather than any postmenopausal changes in women. But nonhormone-mediated effects of genes located on the sex chromosomes may be involved as well.

It is further of note that the pathology leading to ischemic heart disease has a long latency period before it leads to manifest clinical disease and death, and it is, therefore, difficult to interpret momentary cross-sectional associations between sex, age, hormones, and mortality.

The reversal of this sex difference in the transsexual population receiving cross-sex hormones, and the elevated cardiovascular pathology in MtoF, prompted us to review the role of sex steroids (their molecular structures, dose and mode of administration, and age of commencement of cross-sex hormone administration) with regard to their potential role in the elevated cardiovascular pathology in MtoF. Table 1 presents the characteristics of the commonly used estrogens in MtoF. And, vice versa, the lack of apparent unfavorable cardiovascular effects of testosterone administration to FtoM, at least for the term we have been able to follow these effects.

\section{Cross-sex hormone treatment of transsexual persons}

MtoF subjects receive treatment with a compound that suppresses the production/action of testosterone (LHRH agonists or antagonists, cyproterone acetate, progestins) accompanied by estrogenic substances. The pharmacological nature of these estrogens and the route of administration may vary considerably. FtoM subjects are treated with testosterone preparations and, if menstrual bleeding does not stop, a progestational compound may be added, which is no longer needed when surgical sex reassignment including hysterectomy and ovariectomy has taken place. Testosterone molecules are unmodified and circulating testosterone in FtoM is, in part, aromatized to estradiol and circulating estradiol levels do not decline substantially compared with pretreatment levels (for review of cross-sex hormone treatment (13)). If long-term exposure

Table 1 Characteristics of commonly used estrogen preparations in male-to-female transsexual people.

\begin{tabular}{|c|c|c|c|}
\hline & $\begin{array}{l}\text { Transdermal } \\
17 \beta \text {-estradiol }\end{array}$ & $\begin{array}{l}\text { Oral 17 } \beta \text {-estra- } \\
\text { diol valerate/ } \\
\text { hemi-hydrate }\end{array}$ & $\begin{array}{c}17 \alpha \text {-ethinyl } \\
\text { estradiol } \\
\text { (oral or vaginal) }\end{array}$ \\
\hline Dose & $50-100 \mu \mathrm{g}$ & $2-4 \mathrm{mg}$ & $50-100 \mu \mathrm{g}$ \\
\hline Biopotency & + & + & +++ \\
\hline $\begin{array}{l}\text { Venous throm- } \\
\text { bosis risk }\end{array}$ & - & - & $\uparrow \uparrow$ \\
\hline Effect on lipids & Neutral & $\begin{array}{l}\text { Neutral to } \\
\text { negative }\end{array}$ & $\begin{array}{l}\text { Very } \\
\text { negative }\end{array}$ \\
\hline Coagulation & - & $\uparrow$ & $\uparrow \uparrow$ \\
\hline Fibrinolysis & - & $\uparrow$ & $\uparrow$ \\
\hline $\begin{array}{l}\text { Inflammatory } \\
\text { markers }\end{array}$ & - & $\uparrow$ & $\uparrow \uparrow$ \\
\hline $\begin{array}{l}\text { Sex hormone- } \\
\text { binding } \\
\text { globulin }\end{array}$ & - & $\uparrow$ & $\uparrow \uparrow$ \\
\hline
\end{tabular}


to sex steroids is a significant factor in the sex difference in cardiovascular disease, it is of note that transsexual persons start their cross-sex hormone treatment usually between 20 and 40 years of age. The clinic in Amsterdam has started providing treatment to transsexual persons in 1975 and, consequently, we have a significant number of subjects on file having had exposure to cross-sex hormones for a substantial part of their adult lives, but populations that can be studied remain relatively small (14).

When initiating sex reassignment treatment, all subjects attending our clinics had agreed that active participation in a research project was subject to their informed consent, but that their anonymized data could potentially be used in future scientific analysis with the provision that data could not be related to an individual person. This procedure was reviewed and approved by the hospital's ethical review board.

\section{Effects of cross-sex hormone treatment on cardiovascular health in transsexual persons}

Cross-sex hormone treatment both improves and impairs several surrogate cardiovascular risk markers in both MtoF and FtoM (see Tables 2 and 3), but it is not yet clear how these changes in surrogate cardiovascular risk markers translate into the clinical endpoints of cardiovascular disease. Over the past years, several studies have also addressed morbidity and mortality of cross-sex hormone therapy in transsexual persons (Tables 4 and 5).

The current evidence largely suggests that administration of cross-sex hormone therapy to MtoF $(15,16)$ is associated with increased cardiovascular risk. Two studies, assessing mortality in transsexual persons, indeed observed an increased cardiovascular mortality in MtoF compared with the general population $(14,17)$. Interestingly, the Kaplan-Meier curve diverged after about 10 years of follow-up, which could explain previous research that observed no higher cardiovascular mortality after on average 9 years of follow-up (15). The observation of increased cardiovascular morbidity and mortality during cross-sex hormone therapy in MtoF subjects is not entirely unexpected in as far as a parallel can be drawn with hormone use in women. The majority of observational studies and randomized controlled trials show an increased risk of venous thrombosis $(18,19)$ and arterial disease (stroke and myocardial infarction) $(20,21)$ among current contraceptive users and older postmenopausal women using hormone replacement therapy. But age might be a significant variable, because use of hormone therapy in younger menopausal women is associated with a decreased cardiovascular morbidity and mortality (22).

Table 2 Short-term changes in metabolic and cardiovascular risk factors in MtoF transsexual persons.

\begin{tabular}{l} 
Outcome variable \\
\hline Body composition \\
Weight \\
Visceral fat \\
Total body fat \\
Insulin metabolism \\
Fasting glucose \\
Fasting insulin \\
Insulin sensitivity \\
Lipid spectrum \\
Total cholesterol \\
LDL cholesterol \\
HDL cholesterol \\
VLDL cholesterol \\
Triglycerides \\
Fish fatty acid (DHA) \\
Other CVD risk factors \\
Heart rate \\
Diastolic blood pressure \\
Systolic blood pressure \\
Arterial stiffness \\
Hemostasis/fibrinolysis \\
Total homocysteine \\
Inflammation markers \\
\hline
\end{tabular}

Observed changes
Increase
Increase
Increase
No effect
Increase
Decrease
No effect
No effect/increase
Increase
No effect
Increase?
Increase
No effect
No effect/increase
No effect/increase
No effect
Increase
Decrease
No effect/increase

\begin{tabular}{l}
\hline References \\
\hline$(28,40,45,66)$ \\
$(45)$ \\
$(28,66)$ \\
$(28,40)$ \\
$(28,40,66)$ \\
$(28,66)$ \\
$(28,45,66)$ \\
$(28) /(66)$ \\
$(28,66)$ \\
$(28)$ \\
$(40,45)$ \\
$(66)$ \\
$(40)$ \\
$(28) /(40)$ \\
$(28) /(40)$ \\
$(40)$ \\
$(22,45)$ \\
$(48)$ \\
$(48) /(66)$ \\
\end{tabular}

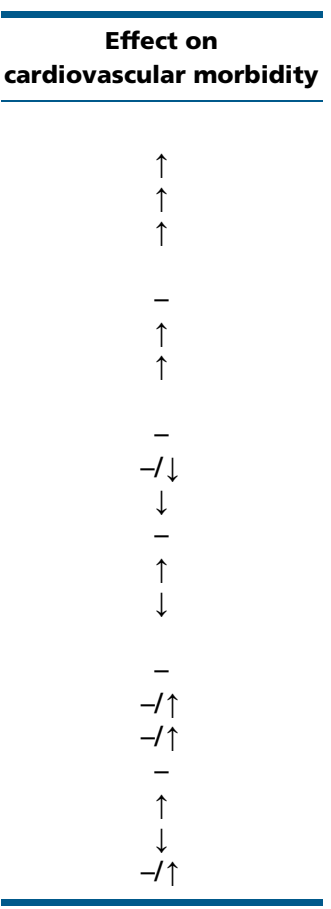

MtoF, male-to-female; DHA, docosahexaenoic acid; CVD, cardiovascular disease. 
Table 3 Short-term changes in metabolic and cardiovascular risk factors in FtoM transsexual persons.

\begin{tabular}{l}
\hline Outcome variable \\
\hline Body composition \\
Weight/BMI \\
Visceral fat \\
Total body fat \\
Insulin metabolism \\
Fasting glucose \\
Fasting insulin \\
Insulin sensitivity \\
Lipid spectrum \\
Total cholesterol \\
LDL cholesterol \\
HDL cholesterol \\
VLDL cholesterol \\
Triglycerides \\
Fish fatty acid (DHA) \\
Other CVD risk factors \\
Heart rate \\
Diastolic blood pressure \\
Systolic blood pressure \\
Arterial stiffness \\
Hemostasis/fibrinolysis \\
Total homocysteine \\
Inflammation markers
\end{tabular}

\begin{tabular}{l} 
Observed changes \\
\hline No effect/increase \\
Slight increase \\
No effect/increase \\
Decrease \\
No effect \\
No effect/slight decrease \\
No effect \\
No effect \\
Decrease \\
No effect \\
Increase \\
Decrease \\
- \\
No effect \\
No effect/increase \\
No effect \\
No effect \\
Increase \\
Increase
\end{tabular}

\begin{tabular}{c}
\hline References \\
\hline$(28) /(40,45,66)$ \\
$(66)$ \\
$(28) /(66)$ \\
$(28,40)$ \\
$(28,40,66)$ \\
$(28) /(66)$ \\
$(28,48,66)$ \\
$(28,40,48,66)$ \\
$(28,40,66)$ \\
$(28)$ \\
$(40,66)$ \\
$(66)$ \\
$(40)$ \\
$(28,40,66)$ \\
$(28,40) /(66)$ \\
$(40)$ \\
$(22,45)$ \\
$(48)$ \\
$(66)$ \\
\end{tabular}

Effect on cardiovascular morbidity

FtoM, female-to-male; DHA, docosahexaenoic acid; CVD, cardiovascular disease.

A detailed study from the gender clinic in Gent (Belgium) reported on cardiovascular disease in 100 transsexual persons (50 MtoF and $50 \mathrm{FtoM}$ ), with an average of 10 years of cross-sex hormone therapy (23). Only three of the MtoF used ethinyl estradiol, and others used transdermal estradiol or oral estrogens (estradiol valerate, estriol). Therefore, it is unlikely that use of ethinyl estradiol has played a significant role in the patterns of elevated cardiovascular pathology in this transsexual population. Three MtoF subjects had experienced thromboembolism (two cerebral and one deep venous thrombosis) during hormone treatment. In addition, in another four MtoF transsexual subjects with other cardiovascular diseases: transient ischemic attack $(n=1)$, venous ulcer $(n=1)$, and myocardial infarction $(n=2)$ were observed. One MtoF subject had a myocardial infarction before hormone therapy (23). Another MtoF underwent surgery for peripheral arterial disease during the course of hormone treatment, but the presence of diabetes mellitus was a likely contributing factor in this person. All participants except one, who experienced thromboembolic or other cardiovascular events, were smokers at the time of event (on average 24 smoking years). In conclusion, cross-sex hormone administration to MtoF, using a variety of estrogens, was associated with a higher degree of cardiovascular morbidity.
The ground-breaking scientific work of BarrettConnor has high-lighted the role of diabetes mellitus as a risk factor in cardiovascular disease, more so for women than for men (24). Women with diabetes mellitus without known heart disease have a similar risk of developing congestive heart failure as nondiabetic women with known heart disease (25). Diabetes confers about a twofold excess risk for a wide range of vascular diseases, independently from other conventional risk factors (26). In both MtoF and FtoM, diabetes mellitus was prevalent in about one-third of the population studied (27). Indeed, one study found that the combination of ethinyl estradiol and cyproterone acetate may induce a degree of insulin resistance in MtoF and be a factor in the development of diabetes mellitus (28) (see also Tables 2 and 3).

In two reports on health status $(15,16)$, analyzing also cardiovascular disease in the transsexual population in the gender clinic in Amsterdam, we were unable to establish an increase in cardiovascular pathology. However, in a report on mortality in the same transsexual subjects published in 2011 (14), we found that ischemic heart disease was the cause of death in $18 \mathrm{MtoF}$ subjects, among the $966 \mathrm{MtoF}$ followed during a mean of 19.4 years of cross-sex hormone treatment. So, it would seem, unsurprisingly, that aging of the same population analyzed in these three studies is a factor in the emergence of cardiovascular disease. Aging 
Table 4 Studies on cardiovascular endpoints in MtoF transsexuals compared with general population or control group.

\section{Reference}

(16)

966

Median duration HRT of 18.5 years* of 4.4 years 9.5 years
Treatment regimen

Ethinyl estradiol $100 \mu \mathrm{g} /$ day and cyproterone acetate $100 \mathrm{mg} /$ day

816 Mean duration HRT of transdermal $17 \beta$-estradio $100 \mu \mathrm{g} /$ twice a week and cyproterone acetate $100 \mathrm{mg} /$ day

Ethinyl estradiol $100 \mu \mathrm{g} /$ day or transdermal $17 \beta$-estradiol $100 \mu \mathrm{g} /$ twice a week and cyproterone acetate $100 \mathrm{mg} /$ day

Median time since SRS of 9.1 years* 58 Mean duration HRT of $6.5 \pm 7.9$ years
Median duration HRT of 6 years
Not specified

Different estrogen regimens and cyproterone acetate $50 \mathrm{mg} /$ day

Different estrogen regimens and cyproterone acetate $50 \mathrm{mg} /$ day median duration of HRT not separately reported for MtoF and MtoF.

\section{Outcome \\ 45-fold increase in VT and/or PE \\ No increased cardiovascular morbidity and mortality \\ 20-fold increase in venous thrombosis and/or pulmonary embolism}

No increased cardiovascular morbidity or mortality rate

Higher mortality due to ischemic heart disease; SMR 1.64 (1.43-1.87)

Higher mortality due to CVD; SMR 2.11 (1.32-3.21) in age group 40-64 years Higher mortality due to cardiovascular disease compared with controls

Lower cardiovascular morbidity compared with control male and female population

Higher number of AMI compared with control women

Higher number of CVD compared with control men and women

HRT, hormone replacement therapy; VT, venous thrombosis; PE, pulmonary embolism; AMI, acute myocardial infarction; CVD, cerebrovascular disease

appeared also to be a factor in the study of Wierckx et al. (27) in the gender clinic in Belgium.

In our previous study (14), the mean age of occurrence of the lethal ischemic cardiac event was 59.7 years (range: 42-79 years). The mean duration of estrogen use was 13.2 years (range: 2-42 years). Eleven of these subjects (61\%) had been using ethinyl estradiol during a mean period of 9.7 years (range: $2-16$ years), whereas the other seven had used transdermal estrogen $(n=2)$, stilbestrol $(n=1)$, tibolone $(n=1)$, or conjugated estrogens $(n=3)$ for a mean period of 16.9 years (range: $5-42$ years). Five MtoF subjects died from stroke. All of them had been using ethinyl estradiol. In the Cox proportional hazard analysis of the type of estrogen treatment in MtoF, current use of ethinyl estradiol was significantly associated with cardiovascular mortality, but not with an increased risk of all-cause mortality or mortality due to other causes. The threefold increased hazard ratio of cardiovascular mortality in current users, compared with never and former users of ethinyl estradiol, remained significant after adjustment for covariates. The fact that only the report of 2011 could establish a significant increased cardiovascular mortality in MtoF indicates that, not surprisingly, there is a time span between use of cross-sex hormones and the manifestation of cardiovascular pathology.
The calculated incidence of cardiovascular mortality was higher in MtoF than in FtoM. The incidence rates were 123 per 100000 person-years in MtoF (95\% CI: 73-173) and 15 per 100000 person-years in FtoM (95\% CI: 1-68).

\section{Parallels of MtoF receiving cross-sex hormones with prostate cancer patients receiving androgen deprivation and/or estrogen treatment}

Androgen deprivation treatment (ADT) is a standard therapy for palliation of metastatic prostate cancer. It is of concern that several retrospective studies have associated ADT with increased risk of diabetes and, possibly, cardiovascular events. Insulin resistance, diabetes, and metabolic syndrome have emerged as the complications of ADT. Some data also suggests that ADT might be responsible for incident cardiovascular disease as found in a populationbased cohort of over 70000 men with prostate cancer with a follow-up of 4.6 years. Treatment with LHRHa was associated with increased risk of incident diabetes by 1.44 (95\% CI: 1.34-1.55), incident coronary heart disease by 1.16 (95\% CI: 1.10-1.21), incident myocardial infarction by 1.11 (95\% CI: 1.01-1.21), and incident sudden cardiac 
Table 5 Studies on cardiovascular endpoints in FtoM transsexuals compared with general population or control population.

\begin{tabular}{|c|c|c|}
\hline Reference & $n$ & Follow-up \\
\hline (16) & 122 & $\begin{array}{l}\text { Median duration HRT } \\
\text { of } 4.4 \text { years }^{a}\end{array}$ \\
\hline (15) & 293 & $\begin{array}{l}\text { Mean duration HRT } \\
\text { of } 8.2 \text { years }\end{array}$ \\
\hline (14) & 365 & $\begin{array}{l}\text { Median duration HRT } \\
\text { of } 18.5 \text { years }^{a}\end{array}$ \\
\hline (17) & 133 & $\begin{array}{l}\text { Median time since SRS } \\
\text { was } 9.1 \text { years }^{a}\end{array}$ \\
\hline (56) & 37 & $\begin{array}{c}\text { Mean duration HRT } \\
\text { of } 4.9 \pm 4.6 \text { years }\end{array}$ \\
\hline (27) & 138 & $\begin{array}{l}\text { Median duration HRT } \\
\text { of } 6 \text { years }\end{array}$ \\
\hline
\end{tabular}

\begin{tabular}{l} 
Treatment regimen \\
\hline Testosterone esters $250 \mathrm{mg}$ i.m. \\
every 2 weeks or testosterone \\
undecanoate $120-160 \mathrm{mg} / \mathrm{day}$ \\
Testosterone esters $250 \mathrm{mg}$ i.m. \\
every 2 weeks or testosterone \\
undecanoate $160 \mathrm{mg} /$ day \\
Testosterone esters $250 \mathrm{mg}$ i.m. \\
every 2 weeks or testosterone \\
undecanoate $160 \mathrm{mg} /$ day \\
Not specified \\
Different testosterone \\
preparations \\
Different testosterone \\
preparations
\end{tabular}

Outcome
No increased cardiovascular morbidity
No increased cardiovascular morbidity
or mortality rate
No increased cardiovascular
mortality rate
Higher mortality due to cardiovascular
disease compared with controls
No difference in cardiovascular
morbidity compared with control
men and women
No difference in cardiovascular
morbidity compared with control
men and women

HRT, hormone replacement therapy.

${ }^{a}$ Median duration of HRT not separately reported for MtoF and MtoF.

death by 1.16 (95\% CI: 1.05-1.27) (29). Similar results have been reported (30), including a review (31).

MtoF people undergo ADT and also receive estrogen treatment. However, this is also the case in some patients with prostate cancer. In a recent study (32), patients were randomly assigned to receive LHRHa or estrogen patches. At 3 months, 93\% receiving LHRHa and 92\% receiving estrogen had achieved castrate testosterone concentrations. After a median follow-up of 19 months, 24 cardiovascular events were reported, six events in six (7.1\%) men in the LHRHa group (95\% CI: 2.7-14.9) and 18 events in 17 (10.1\%) men in the estrogen-patch group (95\% CI: 6.0-15.6). Nine (50\%) of 18 events in the estrogen group occurred after crossover to LHRHa. Mean 12-month changes in fasting glucose concentrations were $0.33 \mathrm{mmol} / \mathrm{l}(5.5 \%)$ in the LHRHa group and $-0.16 \mathrm{mmol} / \mathrm{l}(-2.4 \%)$ in the estrogen-patch group $(P=0.004)$, and for fasting cholesterol were $0.20 \mathrm{mmol} / \mathrm{l}$ $(4.1 \%)$ and $-0.23 \mathrm{mmol} / \mathrm{l} \quad(-3.3 \%)$ respectively $(P<0.0001)$. The results show that parenteral estrogen administered via patches can lead to castrate testosterone concentrations similar to those achieved with LHRHa in men with prostate cancer. In this study, a significant point was that patients with high baseline risks of cardiovascular events were excluded. The rate of cardiovascular complications in men receiving estrogen patches was similar to that in men receiving LHRHa. In addition, it was lower than rates observed with oral estrogen by the Veterans Administration Cooperative Urological Research Group using (oral) diethylstilbestrol (33).
A meta-analysis provided reassuring results. Twenty trials were included in the review. The trials differed with regard to the included patients, formulation and dose of parenteral estrogen, comparator used, outcome measures reported, and the duration of follow-up. The results provide no evidence to suggest that parenteral estrogen is consistently associated with an increase in cardiovascular mortality compare with orchidectomy or LHRHa (34).

There is indeed a difference between metabolic effects of oral and parenteral estrogens; although oral estrogen therapy is known to be associated with thromboembolic complications, studies of parenteral estrogen in men with prostate cancer suggest that the use of parenteral estrogen achieves target androgen suppression, does not adversely affect prothrombotic protein levels, and is not associated with adverse metabolic, skeletal, and body compositional changes when compared with conventional ADT (35).

Oral estrogens were the treatment of choice for carcinoma of the prostate for over 4 decades, but were abandoned because of an excess of cardiovascular and thromboembolic toxicity (33). It is now recognized that most of this toxicity is related to the first-pass portal circulation, which upregulates the hepatic metabolism of hormones, lipids, and coagulation proteins. Most of this toxicity can be avoided by parenteral (i.m. or transdermal) administration of estrogen, which avoids hepatic enzyme induction. It also seems that a short-term but modest increase in cardiovascular morbidity (but not mortality) is compensated for by a long-term cardioprotective 
benefit, which accrues progressively as vascular remodeling develops over time (36). But this view has to be nuanced if pretreatment cardiovascular morbidity is included in the analysis. Patients with previous cardiovascular disease are at considerable risk of cardiovascular events during treatment with high-dose estrogen polyestradiol phosphate (PEP) and even during complete androgen deprivation therapy. Patients without pretreatment cardiovascular morbidity have a moderate cardiovascular risk during estrogen PEP treatment and could be considered for this treatment (37). Also in the study of Wierckx et al. (27) the presence of one or more cardiovascular risk factors predicted cardiovascular disease.

In patients with locally advanced prostatic cancer, PEP estrogen therapy is associated with a statistically significant higher risk of cardiovascular complications compared with orchidectomy (38). The relative risk of diabetes mellitus is increased in men treated with LHRHa by $44 \%$ and the relative risk of cardiovascular morbidity by $10-20 \%$. However, on balance it would seem that estrogen treatment of men with prostate cancer is safe in terms of absolute risk as long as they do not have cardiovascular pathology, but men with cardiovascular pathology may run a clinically important risk.

\section{Relevance of chemical structure and route of estrogen administration to MtoF subjects}

\section{The special role of ethinyl estradiol}

As ethinyl estradiol stood out as a risk factor in MtoF in the study on mortality (14), we have reviewed our earlier studies of the effects of cross-sex hormone administration on surrogate cardiovascular risks (28, 39, 40, 41, 42, 43, 44, $45)$ included in Tables 2 and 3. The estrogen used in our own studies was ethinyl estradiol. The impression is given that the combination of cyproterone acetate + ethinyl estradiol in MtoF had more deleterious effects on variables of cardiovascular risk when compared with testosterone in FtoM. The deleterious effects of ethinyl estradiol are not only the result of its oral administration and its first-pass hepatic effect, but are also due to its long half-life and very high biopotency compared with $17 \beta$-estradiol (46).

Four studies have been conducted in the Amsterdam clinic comparing the effects of oral ethinyl estradiol with transdermal $17 \beta$-estradiol. One study showed that oral ethinyl estradiol treatment of MtoF (compared with transdermal 17 $\beta$-estradiol) was associated with adverse changes in activated protein $\mathrm{C}$ resistance and plasma levels of protein $\mathrm{S}$ and $\mathrm{C}$, while testosterone had antithrombotic effects in FtoM (44). The other study found that administration of oral ethinyl estradiol, but not transdermal $17 \beta$-estradiol, lowered tissue-type plasminogen activator levels in humans without affecting endothelial synthesis (47). The third study found that both oral ethinyl estradiol and transdermal $17 \beta$-estradiol lowered plasma total homocysteine in MtoF $(39,48)$. Finally, oral ethinyl estradiol but not transdermal 17ß-estradiol increased plasma levels of the acute-phase reactant C-reactive protein (CRP). Remarkably, testosterone administration also increased CRP levels in FtoM (49).

Alarmed by the high prevalence of venous thrombosis in MtoF receiving treatment with cyproterone acetate and various types of estrogens, we undertook a study in 2003 which showed that ethinyl estradiol has much stronger prothrombotic effects than oral estrogen preparation such as estradiol valerate or transdermal estrogens (44). This was a reason to halt further administration of ethinyl estradiol to all transsexual subjects under our care. Some transsexual persons, however, continued ethinyl estradiol at their own initiative, receiving prescriptions from other health care providers.

The commonly prescribed dose of ethinyl estradiol to MtoF was $100 \mu \mathrm{g} /$ day, sometimes lowered to $50 \mu \mathrm{g} /$ day after sex reassignment surgery. The ethinyl estradiol content of oral contraceptives has gone down over time from $50 \mu \mathrm{g} /$ day to 30 to $20 \mu \mathrm{g} /$ day. A recent large study on thrombotic stroke and myocardial infarction with oral contraception has concluded that although the absolute risks of thrombotic stroke and myocardial infarction associated with the use of hormonal contraception were low, the risk was increased by $0.9-1.7$ with oral contraceptives that contained ethinyl estradiol at a dose of $20 \mu \mathrm{g} /$ day and by a factor of 1.3-2.3 with those that contained ethinyl estradiol at a dose of 30-40 $\mu \mathrm{g} /$ day, with relatively small differences in risk according to progestin type (50). Both the risks of thrombotic stroke and myocardial infarction were increased. This suggests that there is a dose-response effect, and that MtoF transsexual persons using 50-100 $\mu \mathrm{g}$ are at higher risk of cardiovascular disease than women using modern oral contraceptives with 20-30 $\mu$ g ethinyl estradiol. With other types of estrogens available, it should therefore be strongly recommended against the use of ethinyl estradiol in MtoF subjects who may use estrogens over several decades, which increases both venous and arterial thrombotic events. Adverse effects of ethinyl estradiol on cardiovascular risk are likely due to its prothrombotic, dyslipidemia-inducing and proinflammatory actions (46). There are safer alternatives. 


\section{Route of administration of estrogens}

While ethinyl estradiol has its own typical deleterious effect on cardiovascular risks (due to its chemical profile rather than oral administration), route of administration of $17 \beta$ estradiol is a factor to be considered. In the literature on oral contraceptives, it has been well documented that the type of estrogen and its route of administration has an impact on risk factors of venous thrombosis and cardiovascular disease. This issue has also been addressed earlier in the section on observations in prostate cancer patients treated with estrogens. Oral estradiol increases the markers of fibrinolytic activity and induces potentially antiatherogenic changes in lipids and lipoproteins but also changes markers of coagulation toward hypercoagulability and increases serum CRP concentrations. Transdermal estradiol has no effects on any of these parameters (51). Ethinyl estradiol, compared with $17 \beta$-estradiol, is much more resistant to metabolic degradation and has less favorable effects on lipids, hepatic proteins, angiotensinogen, and on the markers of hemostasis (52). Two studies in transsexual populations indicate that MtoF treated with ethinyl estradiol, compared with other types of estrogen, are more prone to venous thrombosis (44) and to cardiovascular mortality (14). The effects of various types of estrogens and their route of administration in transsexual persons have been excellently reviewed (53).

\section{The effects of testosterone administration on cardiovascular morbidity and mortality in FtoM persons}

Testosterone administration to FtoM affected some cardiovascular risk factors negatively (Table 2) but, notably, there was no induction of insulin resistance, which is the characteristic of hyperandrogenic conditions in women, such as polycystic ovarian syndrome (54). In comparison with certain estrogenic compounds, testosterone is chemically unmodified and administered through non-oral routes (injections or transdermal). There is little information on the oral testosterone undecanoate which has become obsolete because of the low serum testosterone levels achieved.

In a clinical study from Belgium, a substantial degree of cardiovascular risk factors was encountered in FtoM receiving testosterone. Hypercholesterolemia (cholesterol $\geq 190 \mathrm{mg} / \mathrm{dl}$ or $>4.9 \mathrm{mmol} / \mathrm{l}$ ) was observed in $64 \%$ of FtoM. Serum triglycerides were significantly higher (55) in FtoM than in MtoF. A similar number of MtoF and FtoM had an elevated blood pressure at the time of investigation and/or used antihypertensive medication (26 vs $28 \%$ ). But both systolic and diastolic blood pressure were significantly higher in FtoM than in MtoF. None of the FtoM had experienced cardiovascular events such as myocardial infarction, cerebrovascular disease, or deep venous thrombosis.

In other studies of FtoM transsexual persons, a lower or similar cardiovascular morbidity was observed compared with a control population $(15,16,56)$, although one study (17) found a higher cardiovascular mortality rate in FtoM whereas another, in a larger population with a longer term follow-up, did not confirm this (14). Most of the evidence suggests that testosterone treatment in FtoM is relatively safe at short and medium follow-up, although it should be noted that outcome studies in transsexual men are carried out in smaller sample sizes and at significantly younger age of FtoM compared with MtoF. Future research will show whether a parallel can be drawn with men receiving testosterone treatment and who showed an increased risk of a cardiovascularrelated event (55). Our data collection is not large enough and the design of the studies not suited to arrive at a firm conclusion, but the impression is given that exposure of FtoM to testosterone over significant periods of their lifetime is not associated with a strong increase in cardiovascular events. Hyperandrogenic women with the polycystic ovarian syndrome have an established increased risk of developing type 2 diabetes (57) and many cardiovascular risks (58) but a still debated increased risk of cardiovascular disease (59). It is still not clear why, in view of the many cardiovascular risk factors, there is no overwhelming increase in cardiovascular morbidity and mortality in women with the polycystic ovarian syndrome (60). In a study comparing testosterone-treated FtoM with hyperandrogenic women with polycystic ovarian syndrome, we found that testosterone administration induced a decline in serum HDL and increase in triglycerides, but had no effect on insulin resistance, a frequent feature of PCOS (54). Remarkably, cohort studies of premenopausal women followed through the menopause transition suggest that women with oophorectomy are at greater risk for coronary heart disease than intact women, pointing to a greater risk from deficiency of testosterone produced by the postmenopausal ovary than from reduced postmenopausal estradiol levels (61). In summary, it is difficult to establish definitively negative effects of testosterone on cardiovascular disease in females.

In our evaluation of effects of cross-sex hormones on cardiovascular risk, we assessed effects studied in adult life, but the potentiality of prenatal/neonatal factors may be considered as well $(62,63)$. Males are subjected to testosterone exposure prenatally at the time of the formation of the genitalia and again perinatally. FtoM have not had these early exposures. 
There is further a timing hypothesis: differential effects of sex steroids on early and later stages of atherosclerotic disease. Atherosclerosis is characterized by the gradual loss of vascular protective mechanisms and the emergence of advanced, unstable lesions (64). Effects of sex steroids on the endothelium and its protective functions, vascular smooth muscle cells, and inflammatory cells differ depending on the stage of atherosclerosis in the underlying blood vessel.

In addition, not all cardiovascular sex differences are related to differences in sex steroid milieu. In a sex steroid-free environment, there are intrinsic sex-related differences in gene expression and cellular phenotype by microvascular endothelial cells. These intrinsic cell-sex specifics likely contribute significantly to sexual dimorphism in cardiovascular function (65).

\section{Conclusions}

The present analysis does not permit firm conclusions as to the unexpected higher rate of cardiovascular morbidity and mortality in MtoF persons compared with FtoM persons, showing a contrast with the finding of a higher prevalence of cardiovascular disease in men than in women in the general population. Nonetheless, a few speculations can be reasonably offered:

1) In the Amsterdam clinic, increased mortality becomes only apparent in the third-wave analysis. The analyses of 1989 (16) and 1997 (15) had not shown an increase in cardiovascular disease, but the analysis of 2011 (14) clearly produced evidence of increased cardiovascular mortality, so a longer follow-up and aging of the transsexual population - increasing statistical power to analyze cardiovascular endpoints - was in all likelihood a factor, also observed by Wierckx et al. (27).

2) Our studies on the effects of cardiovascular risk factors in the transsexual population (66) and the study with mortality as an endpoint (14) clearly singled out oral ethinyl estradiol as a culprit of the increased rate of cardiovascular pathology. So, this estrogen should no longer be prescribed to MtoF subjects.

3) MtoF subjects are treated with androgen ablation and receive estrogen administration. It is not unreasonable to draw a parallel with men with prostate cancer who receive similar treatment, though transsexual people are considerably younger when they start treatment. In men with prostate cancer, it has appeared that parenteral estrogens are safer with regard to the development of cardiovascular disease (32). Further, estrogen administration was much safer in patients who had no pre-existing cardiovascular disease, at least in terms of absolute risks (37). The available data in MtoF do not allow to determine whether this applies to the MtoF population, but it is not improbable and this was also encountered in the study of Wierckx et al. (27).

4) Diabetes mellitus in women adds considerably to the risk of cardiovascular disease $(24,25)$. Diabetes was often encountered in the study population of MtoF and FtoM in the study of Wierckx et al. (27).

5) It is remarkable that women with hyperandrogenism have a high number of surrogate cardiovascular risk factors, but there is limited convincing evidence that high endogenous or exogenous androgens increase cardiovascular pathology (60). In FtoM, testosterone administration does not induce hyperinsulinism (54). So, if a parallel can be drawn with hyperandrogenic women, it may explain that cardiovascular pathology in FtoM is not significantly increased in most studies. However, more studies are necessary with longer follow-up periods as cohorts of FtoM were relatively small to draw firm conclusions from.

\section{Declaration of interest}

The authors declare that there is no conflict of interest that could be perceived as prejudicing the impartiality of the research reported.

\section{Funding}

L J Gooren received support from 'Stichting Reproductieve Geneeskunde', Amsterdam, The Netherlands. K Wierckx and E J Giltay did not receive any specific grant from any funding agency in the public, commercial, or notfor-profit sector.

\section{Author contribution statement}

All authors, having served as authors of a large number of papers cited here, have made significant contributions to the research of this paper. L J Gooren was leading the draft of the paper which was carefully scrutinized by the other authors.

\section{References}

1 Cooch E, Lank D, Robertson R \& Cooke F. Effects of parental age and environmental change on offspring sex ratio in a precocial bird. Journal of Animal Ecology 199766 189-202. (doi:10.2307/6021)

2 Committee on understanding the biology of sex and gender differences. Exploring the biological contributions to human health. Does sex matter? Eds TM Wizemann \& M-L Pardue. Board of Health Sciences Policy. Institute of Medicine, National Academy Press, Washington, DC, 2001.

3 Weden MM \& Brown RA. Historical and life course timing of the male mortality disadvantage in Europe: epidemiologic transitions, evolution, and behavior. Social Biology 200653 61-80.

4 Horiuchi S. Postmenopausal acceleration of age-related mortality increase. Journals of Gerontology. Series A, Biological Sciences and Medical Sciences 199752 B78-B92. (doi:10.1093/gerona/52A.1.B78) 
5 Trovato F \& Lalu NM. Contribution of cause-specific mortality to changing sex differences in life expectancy: seven nations case study. Social Biology 199845 1-20.

6 Waldron I. What do we know about causes of sex differences in mortality? A review of the literature. Population Bulletin of the United Nations 198518 59-76.

7 Furman RH. Are gonadal hormones (estrogens and androgens) of significance in the development of ischemic heart disease. Annals of the New York Academy of Sciences 1968149 822-833. (doi:10.1111/ j.1749-6632.1968.tb53838.x)

8 Kalin MF \& Zumoff B. Sex hormones and coronary disease: a review of the clinical studies. Steroids 199055 330-352. (doi:10.1016/ 0039-128X(90)90058-J)

9 Haring R, John U, Volzke H, Nauck M, Dorr M, Felix SB \& Wallaschofski $\mathrm{H}$. Low testosterone concentrations in men contribute to the gender gap in cardiovascular morbidity and mortality. Gender Medicine 20129 557-568. (doi:10.1016/j.genm.2012.10.007)

10 Barrett-Connor E. Sex differences in coronary heart disease. Why are women so superior? The 1995 Ancel Keys Lecture. Circulation 199795 252-264. (doi:10.1161/01.CIR.95.1.252)

11 Barrett-Connor E. Clinical review 162: cardiovascular endocrinology 3: an epidemiologist looks at hormones and heart disease in women. Journal of Clinical Endocrinology and Metabolism 200388 4031-4042. (doi:10.1210/jc.2003-030876)

12 Pike MC, Spicer DV, Dahmoush L \& Press MF. Estrogens, progestogens, normal breast cell proliferation, and breast cancer risk. Epidemiologic Reviews 199315 17-35.

13 Hembree WC, Cohen-Kettenis P, Delemarre-van de Waal HA, Gooren LJ, Meyer WJ III, Spack NP, Tangpricha V \& Montori VM. Endocrine treatment of transsexual persons: an Endocrine Society clinical practice guideline. Journal of Clinical Endocrinology and Metabolism 200994 3132-3154. (doi:10.1210/jc.2009-0345)

14 Asscheman H, Giltay EJ, Megens JA, de Ronde WP, van Trotsenburg MA $\&$ Gooren LJ. A long-term follow-up study of mortality in transsexuals receiving treatment with cross-sex hormones. European Journal of Endocrinology 2011164 635-642. (doi:10.1530/EJE-10-1038)

15 Van Kesteren PJ, Asscheman H, Megens JA \& Gooren LJ. Mortality and morbidity in transsexual subjects treated with cross-sex hormones. Clinical Endocrinology 199747 337-342. (doi:10.1046/j.1365-2265.1997.2601068.x)

16 Asscheman H, Gooren LJ \& Eklund PL. Mortality and morbidity in transsexual patients with cross-gender hormone treatment. Metabolism 198938 869-873. (doi:10.1016/0026-0495(89)90233-3)

17 Dhejne C, Lichtenstein P, Boman M, Johansson AL, Langstrom N \& Landen M. Long-term follow-up of transsexual persons undergoing sex reassignment surgery: cohort study in Sweden. PLOS ONE 20116 e16885. (doi:10.1371/journal.pone.0016885)

18 Nelson HD, Humphrey LL, Nygren P, Teutsch SM \& Allan JD. Postmenopausal hormone replacement therapy: scientific review. Journal of the American Medical Association 2002288 872-881. (doi:10.1001/jama.288.7.872)

19 Venous thromboembolic disease and combined oral contraceptives: results of international multicentre case-control study. World Health Organization Collaborative Study of Cardiovascular Disease and Steroid Hormone Contraception. Lancet 1995346 1575-1582. (doi:10.1016/S0140-6736(95)91926-0)

20 Sare GM, Gray LJ \& Bath PM. Association between hormone replacement therapy and subsequent arterial and venous vascular events: a meta-analysis. European Heart Journal 200829 2031-2041. (doi:10.1093/eurheartj/ehn299)

21 Gillum LA, Mamidipudi SK \& Johnston SC. Ischemic stroke risk with oral contraceptives: a meta-analysis. Journal of the American Medical Association 2000284 72-78. (doi:10.1001/jama.284.1.72)

22 Schierbeck LL, Rejnmark L, Tofteng CL, Stilgren L, Eiken P, Mosekilde L, Kober L \& Jensen JE. Effect of hormone replacement therapy on cardiovascular events in recently postmenopausal women: randomised trial. BMJ 2012345 e6409. (doi:10.1136/bmj.e6409)
23 Wierckx K, Mueller S, Weyers S, Van Caenegem E, Roef G, Heylens G \& $\mathrm{T}^{\prime}$ Sjoen G. Long-term evaluation of cross-sex hormone treatment in transsexual persons. Journal of Sexual Medicine 20129 2641-2651. (doi:10.1111/j.1743-6109.2012.02876.x)

24 Barrett-Connor E. The Rancho Bernardo Study: 40 years studying why women have less heart disease than men and how diabetes modifies women's usual cardiac protection. Global Heart 2013 8. (doi:10.1016/ j.gheart.2012.12.002)

25 Daniels LB, Grady D, Mosca L, Collins P, Mitlak BH, Amewou-Atisso MG, Wenger NK \& Barrett-Connor E. Is diabetes mellitus a heart disease equivalent in women? Results from an international study of postmenopausal women in the Raloxifene Use for the Heart (RUTH) Trial. Circulation: Cardiovascular Quality and Outcomes 20136 164-170. (doi:10.1161/CIRCOUTCOMES.112.966986)

26 Sarwar N, Gao P, Seshasai SR, Gobin R, Kaptoge S, Di Angelantonio E, Ingelsson E, Lawlor DA, Selvin E, Stampfer M et al. Diabetes mellitus, fasting blood glucose concentration, and risk of vascular disease: a collaborative meta-analysis of 102 prospective studies. Lancet 2010375 2215-2222. (doi:10.1016/S0140-6736(10)60484-9)

27 Wierckx K, Elaut E, Declercq E, Heylens G, De Cuypere G, Taes Y, Kaufman JM \& T'Sjoen G. Prevalence of cardiovascular disease and cancer during cross-sex hormone therapy in a large cohort of trans persons: a case-control study. European Journal of Endocrinology 2013 169 471-478. (doi:10.1530/EJE-13-0493)

28 Elbers JM, Giltay EJ, Teerlink T, Scheffer PG, Asscheman H, Seidell JC \& Gooren LJ. Effects of sex steroids on components of the insulin resistance syndrome in transsexual subjects. Clinical Endocrinology 2003 58 562-571. (doi:10.1046/j.1365-2265.2003.01753.x)

29 Keating NL, O'Malley AJ \& Smith MR. Diabetes and cardiovascular disease during androgen deprivation therapy for prostate cancer. Journal of Clinical Oncology 200624 4448-4456. (doi:10.1200/JCO.2006.06.2497)

30 Collins L, Mohammed N, Ahmad T \& Basaria S. Androgen deprivation therapy for prostate cancer: implications for cardiometabolic clinical care. Journal of Endocrinological Investigation 201235 332-339.

31 Hamilton EJ, Gianatti E, Strauss BJ, Wentworth J, Lim-Joon D, Bolton D, Zajac JD \& Grossmann M. Increase in visceral and subcutaneous abdominal fat in men with prostate cancer treated with androgen deprivation therapy. Clinical Endocrinology 201174 377-383. (doi:10.1111/j.1365-2265.2010.03942.x)

32 Langley RE, Cafferty FH, Alhasso AA, Rosen SD, Sundaram SK, Freeman SC, Pollock P, Jinks RC, Godsland IF, Kockelbergh R et al. Cardiovascular outcomes in patients with locally advanced and metastatic prostate cancer treated with luteinising-hormone-releasinghormone agonists or transdermal oestrogen: the randomised, phase 2 MRC PATCH trial (PR09). Lancet Oncology 201314 306-316. (doi:10.1016/S1470-2045(13)70025-1)

33 Byar DP \& Corle DK. Hormone therapy for prostate cancer: results of the Veterans Administration Cooperative Urological Research Group studies. National Cancer Institute Monograph 1988 165-170.

34 Norman G, Dean ME, Langley RE, Hodges ZC, Ritchie G, Parmar MK, Sydes MR, Abel P \& Eastwood AJ. Parenteral oestrogen in the treatment of prostate cancer: a systematic review. British Journal of Cancer 200898 697-707. (doi:10.1038/sj.bjc.6604230)

35 Lycette JL, Bland LB, Garzotto M \& Beer TM. Parenteral estrogens for prostate cancer: can a new route of administration overcome old toxicities? Clinical Genitourinary Cancer 20065 198-205. (doi:10.3816/ CGC.2006.n.037)

36 Ockrim J, Lalani el N \& Abel P. Therapy Insight: parenteral estrogen treatment for prostate cancer-a new dawn for an old therapy. Nature Clinical Practice. Oncology 20063 552-563. (doi:10.1038/ncponc0602)

37 Hedlund PO, Johansson R, Damber JE, Hagerman I, Henriksson P, Iversen P, Klarskov P, Mogensen P, Rasmussen F \& Varenhorst E. Significance of pretreatment cardiovascular morbidity as a risk factor during treatment with parenteral oestrogen or combined androgen deprivation of 915 patients with metastasized prostate cancer: evaluation of cardiovascular events in a randomized trial. Scandinavian 
Journal of Urology and Nephrology 201145 346-353. (doi:10.3109/ 00365599.2011.585820)

38 Mikkola A, Aro J, Rannikko S, Oksanen H \& Ruutu M. Cardiovascular complications in patients with advanced prostatic cancer treated by means of orchiectomy or polyestradiol phosphate. Scandinavian Journal of Urology and Nephrology 200539 294-300. (doi:10.1080/ 00365590510031228 )

39 Giltay EJ, Hoogeveen EK, Elbers JM, Gooren LJ, Asscheman H \& Stehouwer CD. Effects of sex steroids on plasma total homocysteine levels: a study in transsexual males and females. Journal of Clinical Endocrinology and Metabolism 199883 550-553. (doi:10.1210/jcem.83.2.4574)

40 Giltay EJ, Lambert J, Gooren LJ, Elbers JM, Steyn M \& Stehouwer CD. Sex steroids, insulin, and arterial stiffness in women and men. Hypertension 199934 590-597. (doi:10.1161/01.HYP.34.4.590)

41 Elbers JM, de Jong S, Teerlink T, Asscheman H, Seidell JC \& Gooren LJ. Changes in fat cell size and in vitro lipolytic activity of abdominal and gluteal adipocytes after a one-year cross-sex hormone administration in transsexuals. Metabolism 199948 1371-1377. (doi:10.1016/ S0026-0495(99)90146-4)

42 Elbers JM, Asscheman H, Seidell JC, Megens JA \& Gooren LJ. Long-term testosterone administration increases visceral fat in female to male transsexuals. Journal of Clinical Endocrinology and Metabolism 1997 82 2044-2047.

43 Bunck MC, Giltay EJ, Diamant M, Gooren LJ \& Teerlink T. Differential effects of cross-sex hormonal treatment on plasma asymmetric dimethylarginine (ADMA) in healthy male-to-female and femaleto-male transsexuals. Atherosclerosis 2009206 245-250. (doi:10.1016/ j.atherosclerosis.2009.01.020)

44 Toorians AW, Thomassen MC, Zweegman S, Magdeleyns EJ, Tans G, Gooren LJ \& Rosing J. Venous thrombosis and changes of hemostatic variables during cross-sex hormone treatment in transsexual people. Journal of Clinical Endocrinology and Metabolism 200388 5723-5729. (doi:10.1210/jc.2003-030520)

45 Giltay EJ, Elbers JM, Gooren LJ, Emeis JJ, Kooistra T, Asscheman H \& Stehouwer CD. Visceral fat accumulation is an important determinant of PAI-1 levels in young, nonobese men and women: modulation by cross-sex hormone administration. Arteriosclerosis, Thrombosis, and Vascular Biology 199818 1716-1722. (doi:10.1161/01.ATV.18.11.1716)

46 Sitruk-Ware R \& Nath A. Characteristics and metabolic effects of estrogen and progestins contained in oral contraceptive pills. Best Practice \& Research. Clinical Endocrinology \& Metabolism 201327 13-24. (doi:10.1016/j.beem.2012.09.004)

47 Giltay EJ, Gooren LJ, Emeis JJ, Kooistra T \& Stehouwer CD. Oral, but not transdermal, administration of estrogens lowers tissue-type plasminogen activator levels in humans without affecting endothelial synthesis. Arteriosclerosis, Thrombosis, and Vascular Biology 200020 1396-1403. (doi:10.1161/01.ATV.20.5.1396)

48 Giltay EJ, Verhoef P, Gooren LJ, Geleijnse JM, Schouten EG \& Stehouwer CD. Oral and transdermal estrogens both lower plasma total homocysteine in male-to-female transsexuals. Atherosclerosis 2003168 139-146. (doi:10.1016/S0021-9150(03)00090-X)

49 Giltay EJ, Gooren LJ, Emeis JJ, Kooistra T \& Stehouwer CD. Oral ethinyl estradiol, but not transdermal $17 \beta$-estradiol, increases plasma C-reactive protein levels in men. Thrombosis and Haemostasis 200084 359-360.

50 Lidegaard O, Lokkegaard E, Jensen A, Skovlund CW \& Keiding N. Thrombotic stroke and myocardial infarction with hormonal contraception. New England Journal of Medicine 2012366 2257-2266. (doi:10.1056/NEJMoa1111840)

51 Vehkavaara S, Silveira A, Hakala-Ala-Pietila T, Virkamaki A, Hovatta O, Hamsten A, Taskinen MR \& Yki-Jarvinen H. Effects of oral and transdermal estrogen replacement therapy on markers of coagulation, fibrinolysis, inflammation and serum lipids and lipoproteins in postmenopausal women. Thrombosis and Haemostasis 200185 619-625.

52 Fruzzetti F, Tremollieres F \& Bitzer J. An overview of the development of combined oral contraceptives containing estradiol: focus on estradiol valerate/dienogest. Gynecological Endocrinology 201228 400-408. (doi:10.3109/09513590.2012.662547)

53 Lioudaki E, Ganotakis ES, Mikhailidis DP \& Nair DR. The estrogenic burden on vascular risk in male-to-female transsexuals. Current Pharmaceutical Design 201016 3815-3822. (doi:10.2174/ 138161210794455049)

54 Cupisti S, Giltay EJ, Gooren LJ, Kronawitter D, Oppelt PG, Beckmann MW, Dittrich R \& Mueller A. The impact of testosterone administration to female-to-male transsexuals on insulin resistance and lipid parameters compared with women with polycystic ovary syndrome. Fertility and Sterility 201094 2647-2653. (doi:10.1016/j. fertnstert.2010.03.048)

55 Xu L, Freeman G, Cowling BJ \& Schooling CM. Testosterone therapy and cardiovascular events among men: a systematic review and metaanalysis of placebo-controlled randomized trials. BMC Medicine 201311 108. (doi:10.1186/1741-7015-11-108)

56 Bazarro-Castro M, Sievers C, Fulda S, Klotsche J, Pieper L, Wittchen H \& Stalla K. Comorbidities in transsexual patients under hormonal treatment compared to age- and gender-matched primary care comparison groups. Reproductive System \& Sexual Disorders 2012. (http:// www.omicsonline.org/2161-038X/2161-038X-1-101.php?aid=3275)

57 Diamanti-Kandarakis E \& Dunaif A. Insulin resistance and the polycystic ovary syndrome revisited: an update on mechanisms and implications. Endocrine Reviews 201233 981-1030. (doi:10.1210/er.2011-1034)

58 Randeva HS, Tan BK, Weickert MO, Lois K, Nestler JE, Sattar N \& Lehnert H. Cardiometabolic aspects of the polycystic ovary syndrome. Endocrine Reviews 201233 812-841. (doi:10.1210/er.2012-1003)

59 Norman RJ, Dewailly D, Legro RS \& Hickey TE. Polycystic ovary syndrome. Lancet 2007370 685-697. (doi:10.1016/S0140-6736(07) 61345-2)

60 Sathyapalan T \& Atkin SL. Recent advances in cardiovascular aspects of polycystic ovary syndrome. European Journal of Endocrinology 2012166 575-583. (doi:10.1530/EJE-11-0755)

61 Barrett-Connor E. Menopause, atherosclerosis, and coronary artery disease. Current Opinion in Pharmacology 201313 186-191. (doi:10.1016/j.coph.2013.01.005)

62 Barker DJ. The developmental origins of adult disease. Journal of the American College of Nutrition 200423 588S-595S. (doi:10.1080/ 07315724.2004.10719428)

63 Chinnathambi V, Balakrishnan M, Yallampalli C \& Sathishkumar K. Prenatal testosterone exposure leads to hypertension that is gonadal hormone-dependent in adult rat male and female offspring. Biology of Reproduction 2012137 131-137. (doi:10.1095/biolreprod.111.097550)

64 Mendelsohn ME \& Karas RH. Molecular and cellular basis of cardiovascular gender differences. Science 2005308 1583-1587. (doi:10.1126/science.1112062)

65 Wang J, Bingaman S \& Huxley VH. Sex differences in white adipose tissue (WAT) and how this may affect the differential responses of males and females to some metabolic challenges. American Journal of Physiology. Heart and Circulatory Physiology 2010298 H1146-H1154. (doi:10.1152/ajpheart.00252.2009)

66 Gooren LJ \& Giltay EJ. Men and women, so different, so similar: observations from cross-sex hormone treatment of transsexual subjects. Andrologia 2014. In press. (doi:10.1111/and.12111)
Received 5 January 2014

Revised version received 4 March 2014

Accepted 10 March 2014 